\title{
Access to Massive Open Online Labs through a MOOC
}

\author{
Wissam Halimi \\ EPFL \\ Lausanne, Switzerland \\ wissam.halimi@epfl.ch

\section{Christophe Salzmann} \\ EPFL \\ Lausanne, Switzerland \\ christophe.salzmann@epfl.ch

\section{Denis Gillet} \\ EPFL \\ Lausanne, Switzerland \\ denis.gillet@epfl.ch
}

Permission to make digital or hard copies of all or part of this work for personal or classroom use is granted without fee provided that copies are not made or distributed for profit or commercial advantage and that copies bear this notice and the full citation on the first page. Copyrights for components of this work owned by others than the author(s) must be honored. Abstracting with credit is permitted. To copy otherwise, or republish, to post on servers or to redistribute to lists, requires prior specific permission and/or a fee. Request permissions from Permissions@acm.org. L@S 2017, April 20-21, 2017, Cambridge, MA, USA.

$L$ (a) 2017 , April Copyright is held by the owner/author(s). Publication rights licensed to ACM ACM $978-1-4503-4450-0 / 1704 \ldots \$ 15.00$.

DOI: http://dx.doi.org/10.1145/3051457.3054017

\begin{abstract}
Few MOOCs offer laboratory work as part of their educational material, yet it is known that hands-on sessions are important components of science and engineering education. Equally important is understanding how students are using labs as part of their learning activity without the constraints of space and time. In this work we present the initial results of the usage of a remote lab provided as part of a Control Systems MOOC.
\end{abstract}

\section{Author Keywords}

MOOC; Massive Open Online Labs; MOOL, online education; remote labs, engineering education.

\section{ACM Classification Keywords}

- Human-centered computing Web-based

interaction - Applied computing Interactive learning environments • Applied computing Distance

learning • Applied computing E-learning

\section{Introduction}

Today MOOCs are a source of affordable and convenient knowledge. Most MOOCs offer knowledge evaluation tools such as quizzes or graded assignments, touching on the practice side of completing a course. But to the extent of our knowledge, very few MOOCs offer laboratory work. It is a given that hands-on sessions are essential for a 


\section{Control Systems MOOC:}

Logistics: At EPFL, the

Control Systems course is

taught in an unconventional way: lectures are given in class, and hands-on sessions available through the MOOC (flipped classroom). In the MOOC, students have lectures summaries and instructions for

experimentation to watch, quizzes to take, and remote access to the physical labs.

Typically, the student opens the MOOC and goes through a sequence of tabs, each tab with relative material to study. There is an allocated and reserved time for students to use the MOOC at the premise, during which teaching assistants are present to answer any questions the students might have, in addition to the continuous MOOC availability.

Remote lab access: The remote lab is integrated in the MOOC in a separate tab than the other material. The user interface is shown in Figure 1 . The students can save their experimental results, and load them in another available tool for system modeling and simulation, see [2]. complete science and engineering education. Therefore, we identify the need to support lab work in MOOCs.

Nowadays there is a surge in remote labs use in blended and distance learning. The main motivations for developing and deploying remote labs are the unbounded accessibility by space and time, the sharing of resources among different institutions, and lowering costs of laboratory ownership, maintenance, and scalability as the number of students increases. In this context, a remote lab is a real physical lab, which is accessible through the Internet at distance.

For more than ten years now, the Automatic Contro Lab at EPFL (Ecole Polytechnique Fédérale de Lausanne) offers its students the ability to remotely connect to the lab of the Control Systems course. And recently, major efforts have been put into deploying the remote lab on a large scale, as part of its new MOOC: Control Systems [2][3].

In this work, we present the remote lab deployed as part of the educational resources of the mentioned MOOC. In order to extract interesting patterns in lab use, we gather activity tracks touching on different dimensions of students' interaction with the remote lab, this would give insight on how to enhance their learning experience and how to scale it for larger number of students thanks to learning analytics. Learning analytics is a major source of insight to infer about learning patterns, identifying what makes a student successful, and often used for personalization and recommendation [1].

\section{The Lab}

The complete laboratory infrastructure services 25 installations of servo drives. At the time of writing, the MOOC is deployed on a local copy of edX hosted by $E P F L$, yet accessible to the world. The total number of students taking the course is around 250 per semester. The course ran from the $20^{\text {th }}$ of September to the $23^{\text {rd }}$ of December 2016. For the exception of some weeks, the students were required to go to the physical lab 2 times per week.

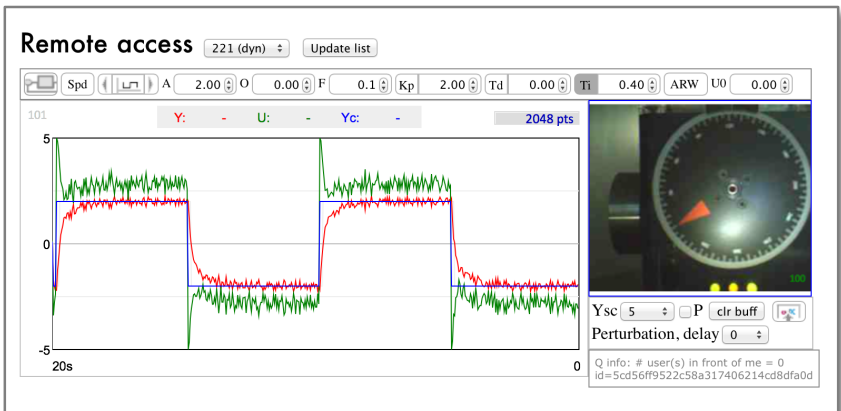

Figure 1: The user interface composed of a command strip through which the students can control the lab, an oscilloscope screen, and a video feed as shown in the MOOC

\section{Data}

For every connection, the lab saves activity tracks from the time of connection to disconnection. The tracks contain information regarding the time of connection, the role (controller who can act on the setup or observer who can watch what the controller is doing and see the results), unique identifiers for location of connection and user, parameters pushed for experimentation, remaining time of experimentation, and other system specific logs for security and keeping 
the lab in a safe state. Over 500000 activity tracks were gathered during the period of the course.

\section{Statistics}

Location of connection: We can see that $10.74 \%$ of the connections were made from the lab room (students can see the equipment). $8.54 \%$ came from the EPFL campus network, $61.16 \%$ from the either the EPFL WiFi network or remote VPN access, and $19.56 \%$ from outside any EPFL provided connection. A considerable portion of the students is returning to the lab from outside the university, mostly between the hours 18:00 and $21: 00$ on the same day as the lab or the next.

Duration of experimentation: To handle queues, one strategy was to allocate to each experiment tab a fixed duration. If the allowed time expires and there are queuing users, the current user is pushed into the queue as an observer. If the allowed experimentation time is expired but there are no waiting users, the current user can keep hold of control. We assume that students who didn't stay connected for more than 10 seconds, are not really using the lab. Depending on the experiments, the maximum allowed time ranged from 90 seconds to 2 minutes per session. Regardless of the fixed allocated time for experimentation and the expectation of the theoretical massive queuing (250 students for 25 setups with dynamical allocation), we found that the minimum connection time is 11 seconds, the maximum is 1 hour, the mean is $6 \mathrm{~min}$ and 22 seconds, with a standard deviation of $11 \mathrm{~min}$ and 29 seconds; hence the system is far from saturation.

Queue Sizes: The maximum queue size encountered by students is $3(0.1 \%) .2$ users queued $2.89 \%$ of the time, and $97 \%$ of connections were for single users.
Interestingly enough, when removing all students who spent less than 10 seconds connected, we don't observe any more queuing, i.e. no more observers and it seems that the 25 setups are successfully servicing the connected users. More specifically, no students waited more than a second in a queue. That is queuing is only happening during the peak time for connections, which is during the lab sessions, and it seems that students who are gaining control of the setup are leading the group work, and others are disconnecting or switching to other tasks.

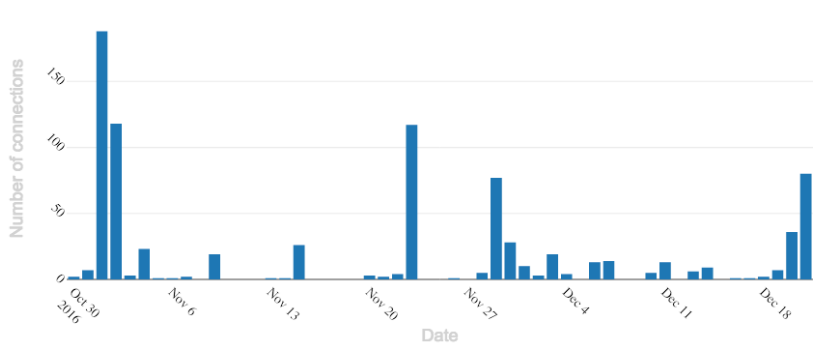

Figure 2: Number of connections throughout the whole period of the course.

\section{Concurrent Access}

In Figure 2, we show all the days during which the students accessed the MOOC. Of the 49 days, on 37 days the lab was used. This shows that students are not only interested in the material offered by the course, but given the opportunity they will take it in order to experiment. Knowing the dates of the lab sessions, we see that students mostly connected to the lab on those days. The maximum number of connections per day is 188 , the minimum is 1 , the mean is 17.75 and the standard deviation is 36.84 . But more importantly, there were more enthusiastic about it during the beginning of the semester, mid-semester, 
and the end of it. Also, they seem to be more eager to use the lab around the time of the midterm: week of the $21^{\text {st }}$ of November, and towards the end of the course.

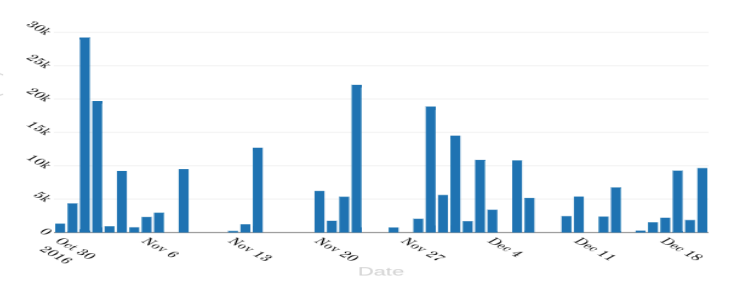

Figure 3: Time spent on experimentation through the whole period of the course

Figure 3 shows the time spent by students experimenting when they connect to the MOOC. In comparison with Figure 2, we see that the number of connections per day does not imply a longer usage time of the lab.

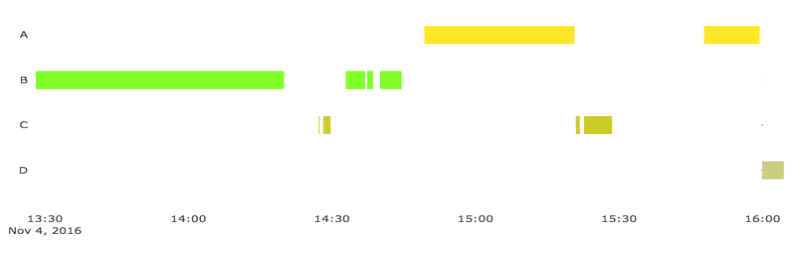

Figure 4: Occupation of one lab setup, outside lab hours, for one of the busiest hours

We isolate the connections made outside the lab session hours from the rest of the activity tracks, and observe the concurrent access behavior for one setup. In Figure 4 we can observe one of the busiest hours where 4 users tried to use the lab. There is no overlap between any of the users, yet the minimum elapsed time between 2 users is less than 30 seconds. This is mainly the result of having multiple setups servicing the requests: if one setup is busy the user is redirected to an available one, and there was never a time where users had to wait for access.

\section{Conclusion and Future Works}

From the initial analysis, we see that a 10:1 ratio is possible for servicing the students with the current configuration (25 setups-250 users). While we cannot clearly differentiate between local Wi-Fi and VPN access, we notice that a large part of the MOOC accesses originates from outside the premise. The average experimentation time of $6 \mathrm{~min}$ is larger than the pre-allocated time, thus this duration could be dynamically set for each experiments. Moreover, the collected data can be utilized to devise access management to the lab given a larger number of users, especially through gamification. At the time of writing, the students hadn't yet taken the final exam, hence the correlation between grade and time spent on the MOOC is yet unknown.

\section{References}

1. Duval, E. Attention please!: learning analytics for visualization and recommendation. In Proceedings of the 1st International Conference on Learning Analytics and knowledge. ACM (2011).

2. Salzmann, C., Halimi, W., Gillet, D., and Govaerts, S. Deploying Large Scale Online Labs with Smart Devices. In Cyber-Physical Laboratories in Engineering and Science Education (In Press).

3. Salzmann, C., Gillet, D., and Piguet, Y. MoOls for MOOCs: A first edX scalable implementation. In The $13^{\text {th }}$ International Conference on Remote Engineering and Virtual Instrumentation. IEEE, 2016. 